Impacto de la era digital en el perfil de comunicación

Verónica García Martínez, Angélica Fabila Echauri, Martha Patricia Silva Payró

Question/Cuestión, Vol. 2, N 66, Agosto 2020

ISSNe 1669-6581

https://perio.unlp.edu.ar/ojs/index.php/question/index

IICom-FPyCS-UNLP

DOI: $\underline{\text { https//doi.org/10.24215/16696581e483 }}$

\section{Impacto de la era digital en el perfil de comunicación}

\section{Impact of the digital age on the communication profile}

Verónica García Martínez

vero1066@hotmail.com

https://orcid.org/0000-0001-5299-3540

Doctora en Ciencias Sociales por la Facultad Latinoamericana de Ciencias Sociales (FLACSO) México. Profesora - investigadora de la Universidad Juárez

Autónoma de Tabasco, en la División Académica de Educación y Artes.

Miembro del Sistema Nacional de Investigadores. Integrante del Cuerpo Académico Consolidado Investigación e Innovación Educativas (PRODEP). Miembro del Consejo Mexicano de Investigación Educativa. Miembro de la Red

LaTe de Conacyt. Miembro de Registro Conacyt de Evaluadores Acreditados (RCEA). Las Áreas de interés en las que desarrolla investigación se centran en

Sociología educativa con énfasis en Tecnología y Educación, sujetos de la universidad.

Universidad Juárez Autónoma de Tabasco - México 


\section{Angélica Fabila Echauri} angelifa@hotmail.com https://orcid.org/0000-0001-5667-3712

Doctora en Comunicación Educativa, por la Universidad de Baja California. Profesora - investigadora de la Universidad Juárez Autónoma de Tabasco, en la División Académica de Educación y Artes. Miembro del Sistema Estatal de Investigadores. Integrante del Cuerpo Académico: Procesos comunicativos y formativos en escenarios emergentes. Las Áreas de interés en las que desarrolla investigación se centran en Procesos emergentes de comunicación; Comunicación educativa y Medios y Tecnología. Universidad Juárez Autónoma de Tabasco - México

\section{Martha Patricia Silva Payró patypayro@gmail.com https://orcid.org/0000-0001-9081-0549} Licenciada en Informática Administrativa por la Universidad Juárez Autónoma de Tabasco (1998), Maestra en Dirección de Empresas por el Instituto Universitario Puebla (2015) y Doctora en Administración Educativa por la Universidad Juárez Autónoma de Tabasco (2018). Es profesora investigadora de la División Académica de Ciencias y Tecnologías de la Información de la UJAT. Miembro del Sistema Nacional de Investigadores de México, nivel 1. Se especializa en internacionalización de la educación e innovación aplicada a la educación y ha publicado diversos artículos y capítulos de libro en Cuba, España, Costa Rica, México y otros países de América Latina. Universidad Juárez Autónoma de Tabasco - México 


\section{Resumen}

El objetivo del estudio fue analizar los cambios que tuvo el currículum a lo largo de su trayectoria como resultado de las constantes innovaciones tecnológicas que han propiciado nuevos perfiles del profesional en Comunicación de una universidad pública del sureste de México. Se presenta el estudio de caso en el cual se realizaron análisis documental y de contenido curricular, así como entrevistas a profundidad con profesores que imparten cátedra desde hace dos décadas en el programa. Los resultados señalan que, tanto en el currículum como desde la perspectiva de los docentes, existe una reconfiguración del campo profesional de la comunicación. Se concluye que hay un proceso circular entre los cambios que demanda el entorno y las modificaciones que ameritan los planes de estudio.

\section{Palabras clave}

Era digital, perfil profesional, currículo, comunicación.

\section{Abstract}

The objective of the study was to analyze the changes that the curriculum had throughout its career as a result of the constant technological innovations that have led to new profiles of the professional in Communication of a public university in southeastern Mexico. The case study is presented, in which documentary and curricular content analyses were carried out, as well as indepth interviews with professors who have been teaching in the program for two decades. The results indicate that, both in the curriculum and from the 
perspective of teachers, there is a reconfiguration of the professional field of communication. It is concluded that there is a circular process between the changes demanded by the environment and the modifications that curricula require.

\section{Key words}

Digital era; professional profile; curriculum; communication.

\section{Introducción}

La digitalización como proceso irrumpe en todos los ámbitos de la vida humana sobre todo en la dimensión social y comporta cambios en las formas de comunicarse. "La comunicación profesional está cambiando dramáticamente en la era digital" (Porter, 2017, p.1), como disciplina y profesión experimenta transformaciones originadas por el imparable avance tecnológico. En los ámbitos donde el comunicólogo se mueve se eliminan, crean y modifican los roles tradicionalmente asignados a un profesional formado en las filas universitarias: medios de comunicación, agencias de publicidad, organizaciones y otros espacios que demandan servicios comunicacionales.

La digitalización es caracterizada según Scolari, Mico, Navarro y Pardo (2008) por la aparición de soportes textuales y dispositivos de producción y distribución basados en código binario, intercambios de información a través de redes, nuevas formas de organización en la producción, convergencia de lenguajes y formas nuevas de crear y transmitir datos. No representa solo un cambio de formato, sino que plantea una reconfiguración de los medios en donde el usuario es quien dicta los temas y contenidos (Jódar, 2010).

Pero al igual que los entornos, también los sujetos cambian, las generaciones 
de jóvenes que van protagonizando las olas tecnológicas son etiquetadas de acuerdo a ciertas características que los distinguen en su momento. Molina y López (2018) señalan que en las universidades actuales perviven tres de ellas que representan a la generación Einstein (1988 a la actualidad) y que los vincula el hecho de haber nacido en una época de avances tecnológicos: la de los digitales adaptativos o gen " $X$ " (1965-1979), los nativos digitales, gen "Y" o millenials (1980-2000) y los avatares digitales o nacidos en el siglo XXI.

Este panorama pone en aprietos a las sociedades adultas cuya inserción a la era digital es lenta o nula, y que debe hacer frente a lo que García-Aretio (2019) Ilama las "sacudidas tecnológicas" representadas entre otras cosas por la inteligencia artificial, el internet de las cosas, la realidad mixta, artefactos autónomos, dispositivos inteligentes, big data, blockchain, computación cuántica, y más; puntualiza que los trabajos más demandados hoy, no existían en 2005 y no existirán en el futuro, por lo menos no como los conocemos.

Pese a esta inminente extinción, son muchos los que consideran que este escenario ofrece un cúmulo de posibilidades para la creación de nuevos perfiles, ya que las empresas necesitan profesionales capaces de gestionar nuevas funciones desde una perspectiva digital (Silva-Robles, 2016). Sin embargo, deben desarrollarse competencias que sirvan a los distintos sectores, de modo que la formación deberá ser enfocada (Ventura, Roca-Cuberes y Corral-Rodríguez, 2018), puesto que en cada ámbito (medios, marketing y publicidad, relaciones corporativas etc.) se requiere del manejo de plataformas especializadas de acuerdo a las actividades de las agencias, aunque esta condición paradójicamente crea al profesional polivalente, lo que provoca un proceso de reconversión y en otros de redefinición de sus cometidos (Soler y Aguilar, 2009). 
En este contexto la educación superior se ha visto forzada a adaptarse para tratar de responder a los requerimientos del entorno y asumir la responsabilidad histórica de asimilar los aportes de la revolución tecnológica. Actualmente en las aulas universitarias se encuentran estudiantes profundamente influidos por la vida en línea, la virtualidad, la inmediatez informativa, la multitarea, la predilección de mensajes visuales sobre los textuales, la hipertextualidad, lo lúdico, las identidades digitales, y los aprendizajes extra muros (García-Aretio, 2019).

De ahí que como afirma Brewer (2013) los jóvenes tendrán cada vez más dificultades para encontrar trabajo por el desajuste entre su formación y las exigencias del mercado laboral. Frente a esta realidad las universidades se han visto en la necesidad de replantear sus principios y modelos para proponer cambios curriculares que han sido incorporados durante décadas a los programas educativos, aunque no con la misma celeridad con que transita la era digital.

Partiendo de este marco se plantearon las preguntas ¿cuáles han sido los cambios curriculares propiciados por la era digital en las áreas de formación de la Licenciatura en Comunicación de una universidad pública del sureste mexicano? ¿cómo han asumido los profesores del programa educativo los cambios curriculares derivados del impacto tecnológico? En consonancia con esto se planteó el objetivo de: analizar los cambios curriculares de un programa educativo de Comunicación y la experiencia de los profesores vinculados a éste, derivados de los cambios propiciados por la era digital en la profesión del comunicólogo.

El programa de esta licenciatura se orienta principalmente a tres áreas 
tradicionalmente conocidas en la disciplina: periodismo y divulgación, comunicación organizacional, así como diseño y publicidad. A través del estudio de caso se desarrolla este trabajo. El documento hace un abordaje de los nuevos perfiles del comunicólogo derivados del cambio tecnológico emparejados con las áreas disciplinares de la licenciatura antes mencionadas. En otra sección se describe el método y por último los resultados y conclusiones.

\section{Nuevos perfiles profesionales en el periodismo y los medios audiovisuales}

Las prácticas del periodista desde hace más de dos décadas han ido cambiando radicalmente debido a la tecnología. Los cambios de los que se hablaba hace una década para esta profesión en el futuro ya tienen lugar hoy (Anderson, Bell \& Shirky, 2017). La Internet representó desde su aparición un desafío para el periodista, quien se ha visto en la necesidad de hacer uso de herramientas y estrategias innovadoras para desarrollar un trabajo de calidad (Brandtzaeg, et al, 2015). Este oficio habrá de sintetizar las funciones de búsqueda, grabación, organización, edición y difusión de los mensajes, sean escritos o imágenes para cualquier medio de comunicación, con los diferentes lenguajes y para diversidad de soportes tecnológicos (López, 2012; Cho, Furey \& Mohr, 2016). Además, la condición de freelance será cada vez más frecuente (Meso, 2003; Kerr \& Keheller, 2015).

Además del dominio tecnológico, el periodista digital debe estar atento a las reacciones de los lectores, sobre todo a través de las redes sociales y de los blogs y responder en tiempo real a las audiencias. El oficio puede estar impregnado de los saberes en tecnología, pero no deja de lado los principios 
que lo han definido desde su origen: la veracidad, objetividad, independencia y humanidad, los cuales siguen siendo la base del mismo (López-García, Rodríguez-Vázquez y Pereira-Fariña, 2017). Una de las derivaciones del perfil es el media browser (Scolari et al, 2008), que es un periodista que se encarga de administrar los metadatos recibidos de las agencias y añaden información adicional para elevar su calidad y atracción.

Con relación a la acción del comunicólogo en los medios audiovisuales también se detectan varios nuevos perfiles, el documentalista por ejemplo (Benítez, 2007; Ripoll-Mont y Tolosa-Robledo, 2009) sujeto freelance que también sintetiza varias tareas, pero cuya función principal es la recuperación, acopio, depuración y organización de documentos sobre todo video gráficos anidados en la Internet. Este profesional debe reunir conocimientos para el tratamiento y gestión audiovisual, así como actividades de búsqueda. Este perfil es similar, y algunos hasta lo utilizan indistintamente al llamado gestor de contenidos o media manager (Scolari et al, 2008; Soler y Aguilar, 2009), clave en el almacenaje y borrado del material digitalizado que se encuentra en la red y sirve al proyecto de la organización para la cual trabaja.

Estas figuras son importantes en las emisoras de contenidos (entretenimiento, noticias) donde cobran importancia el texto y la imagen bajo la condición de la inmediatez (Ripoll-Mont y Tolosa-Robledo, 2009), que incluyen tanto televisoras como radiodifusoras, que utilizan sobre todo Internet como plataforma. En este sentido, tanto unas como otras han incursionado a lo digital a través de hardware y software, y ambas utilizan las redes sociales para diversificar su difusión. Señalan Muñoz-de-la-Peña-Costero, Meana-Alonso y Sáez-Carreras (2014, p. 73) que "el nuevo sistema de producción digital ha traído consigo la implantación de un nuevo diseño de gestión de contenidos 
que ha transformado la forma de trabajar y ha cambiado de forma radical la estructura de algunos departamentos".

En esas estructuras, la interactividad con los consumidores es también un elemento imprescindible (Soler-Adillon et al, 2016). En el caso de la radio, por ejemplo, la relación con la audiencia se modifica constantemente, sobre todo porque ahora el espacio público son las redes que requieren de la actualización de esquemas y narrativas con nuevos códigos y etiquetas para interactuar instantáneamente con los oyentes (García y Román, 2018). Ya no es la relación distante entre emisor y receptor sino una más privada y estrecha. Incluso muchas emisoras radiofónicas utilizan algunas plataformas para difundir las imágenes de los presentadores para crear una atmósfera más personal.

En estas estructuras de creación y gestión de contenidos se pueden identificar más de 20 ofertas distribuidas en tres perfiles profesionales (Álvarez-Flores, Núñez-Gómez, Olivares-Santamarina, 2018): director de contenidos, gestor de contenidos digitales y redactor web.

\section{Nuevos perfiles profesionales en comunicación organizacional}

El comunicólogo también debe ser capaz de desenvolverse en el área de la comunicación organizacional que puede implicar a las relaciones públicas internas y externas, además reconocer la identidad corporativa como posibilidad de desarrollo profesional a modo de gestionar la comunicación en las empresas de cualquier giro (Miquel, López y Gil, 2018).

En las relacionas públicas (RP) sean internas o externas se identifican varios tipos de perfiles participantes (Silva-Robles, 2016): los promotores (que facilitan la consecución de objetivos) los ejecutores (que se ocupan de planificar y 
ejecutar las acciones de RP) y los públicos (con los que se desean armonizar sus intereses y visiones). En el perfil tradicional estos sujetos no tenían una interacción tan abierta como lo es ahora con ayuda de los soportes tecnológicos, en donde muchas de las relaciones se dan a través de las plataformas (blogs, redes, páginas web.). Las RP ayudan a generar y mantener audiencias activas, repercutiendo en el índice de engagement online (MartínezSala, y Campillo-Alhama, 2018) y propician la construcción de la identidad en las marcas, aunque siempre con el apoyo de un manejo experto de lenguaje (Cheney \& Ashcraft, 2007).

La web participativa ha cambiado a la comunicación corporativa tanto en forma como en fondo, por lo que el comunicador corporativo debe entrenarse en la manera de escuchar a la audiencia a la que se va a dirigir y utilizar recursos relacionados con la tecnología que le faciliten hacerlo (Molina y López, 2018), pero además debe buscar mantener alta la moral de los empleados de la empresa (DuFrene \& Lehman, 2014). En esta profesión la interactividad es un elemento fundamental, dado que son los clientes quienes determinan a través de su visión los derroteros de una marca (en este sentido se habla de la cocreación).

En este ambiente surge una figura de gran fuerza profesional que se le conoce como community manager (CM), cuya función ya comienza a ser objeto de estudio (Elorriaga-Illera, Usin-Enales, y García-Azpuru 2018; Ortega, Rendón y Ortega 2017; Silva-Robles, 2016), a tal punto que se han identificado tres tipos de CM que no necesariamente deben estar adscritos a la estructura de la organización (Ortega, Rendón y Ortega, 2017): los gestores de comunidades "per se", los gestores de comunidades generadas por marcas y los gestores de diferentes comunidades de diferentes marcas. Ortega, Rendón y Ortega (2017) 
señalan que el CM debe ser formado como geek (apasionado por la tecnología e informática), early adopter (que valida y recomienda un producto o servicio) o influencer (prescriptor de una marca).

Los profesionales que actualmente están desarrollando este perfil pertenecen a diferentes formaciones disciplinares, pero especialmente a carreras de periodismo y comunicación (Kerr \& Keheller, 2015; Elorriaga-Illera, UsinEnales, y García-Azpuru 2018; Miquel, López y Gil, 2018) y tienen además de un amplio espectro de habilidades informáticas y telemáticas dominio de idiomas, especialmente inglés.

\section{Nuevos perfiles profesionales en diseño y publicidad}

Otra área de acción del comunicólogo está relacionada con el diseño y publicidad. En estos tiempos se habla del diseñador de interactivos (Ddl) (Soler-Adillon et al, 2016) más cercano a las artes y humanidades y que desarrolla creaciones digitales para establecer una comunicación interactiva con las audiencias y más cercano a la praxis que a la teoría. En el diseño de interacción destacan tres ámbitos de influencia: interacción persona-ordenador, diseño y creación digital (Soler-Adillon et al, 2016, p.199). Este perfil demanda de la sintetización de saberes entre la ingeniería y arquitectura y la humanística, con una alta adaptabilidad a los cambios tecnológicos. El diseño gráfico es un elemento que también es modificado continuamente por el contexto tecnológico a través de un gran número de programas orientados a la comunicación audiovisual.

En esta área destacan: el diseñador publicitario, el director de arte, el diseñador web y el desarrollador web (Álvarez-Flores, Núñez-Gómez, OlivaresSantamarina, 2018), que se dedican a la construcción de conceptos creativos 
para diversos formatos y plataformas (banners, webs, emailing, landing pages etc.). Para esta profesión, aparte del manejo de software de diseño y edición de video se debe manejar la maquetación web y el inglés. Aunque el diseño existe por sí mismo, en realidad es creado con un propósito publicitario, por lo que indistintamente (aunque no necesariamente) ambos conceptos se vinculan en el ámbito de la comunicación.

La publicidad es una actividad trastocada por la tecnología, que la ha empujado a convertirse en un servicio personalizado en tiempo real, y que le ofrece a cada usuario lo que necesita y de este modo optimiza la inversión del anunciante (Carrillo-Durán y Rodríguez-Silgado, 2018). Las diversas plataformas y redes sociales montadas en internet han modificado la forma en que las empresas usan la publicidad (Jameson, 2014). Los viejos medios, aunque no desaparecen, cambian los contenidos publicitarios y diversifican sus canales apoyados hoy en la internet, creando cadenas de valor con los consumidores (Rowley, 2008). De la misma manera, las redes sociales le dan al usuario y/o consumidor la posibilidad de convertirse en interlocutor o incluso en anunciante. Advierten Carrillo-Durán y Rodríguez-Silgado (2018, p. 200) que "El reto es que los nuevos profesionales no sólo deberán tener conocimientos de marketing y comunicación sino también de programación y gestión de datos, dejando al margen la aparición de perfiles relacionados directamente con la informática o herederos de los perfiles tradicionales".

Como se advierte en la revisión de la literatura, existen elementos recurrentes en los perfiles analizados, pero pueden destacarse el uso de la internet y especialmente de las redes sociales como elemento transportador de doble vía entre las organizaciones o agencias y los consumidores. Diversos estudios muestran que las redes, son los espacios o herramientas imprescindibles para 
el nuevo perfil profesional y se convierten en una constante para las estrategias de marketing, comunicación y de relaciones públicas (Martínez-Sala y Campillo-Alhama, 2018; Meso, 2003).

\section{Método}

La investigación se desarrolló desde el enfoque metodológico cualitativo, con el propósito de indagar sobre la perspectiva detallada de los participantes, buscando capturar sus voces y permitiendo que sus experiencias fueran entendidas en el contexto del estudio (Bazeley, 2013, p. 27; Creswell, 2015, p.5).

El método utilizado fue el estudio de caso que, como señala Yin (2012, p. 4), se utiliza en muchas situaciones para contribuir al conocimiento de fenómenos individuales, grupales, organizativos, sociales y políticos. Para el caso de este estudio, se empleó la revisión de documentos institucionales, así como entrevistas a profundidad, las cuales se audio grabaron. Éstas permitieron un intercambio adecuado de información entre entrevistador y entrevistado en el transcurso de la misma (Edwards y Holland, 2013, p. 78).

Se obtuvo autorización de las autoridades universitarias para la realización del estudio. Se solicitó el consentimiento informado de los profesores para participar en el estudio. Se les garantizó confidencialidad en el manejo de la información que proporcionaron.

Las técnicas para la recopilación de información que se emplearon, bajo una estrategia de complementación, fueron el análisis curricular comparado y la entrevista a profundidad con los profesores. Esta consideró dos preguntas críticas: ¿Cuál es el impacto que la era digital ha tenido en la formación del comunicólogo? ¿Cómo ha tratado el cambio curricular en esta licenciatura de 
responder a las implicaciones del cambio tecnológico?

En las entrevistas participaron dos profesores y dos profesoras de la Licenciatura en Comunicación, que han transitado en al menos tres de los cuatro cambios curriculares que el Plan de Estudios ha tenido. Entre ellos una profesora fundadora de la licenciatura. Todos con participación activa en la toma de decisiones en los procesos de reestructuración de los planes y programas de estudio. Se efectuó la audio grabación de las entrevistas con la autorización de los entrevistados y posteriormente, para su análisis, fue transcrito en versión extensa. La organización y sistematización de la información recabada con las entrevistas se llevó a cabo mediante la formulación de matrices de análisis de contenido.

Para darle validez y credibilidad varios investigadores participaron en la realización y el análisis de las entrevistas. Para una comprobación de coherencia investigadores externos identificaron en los textos las categorías de análisis generadas en el estudio. Finalmente, se realizó un control de credibilidad al presentarles y discutir con los académicos involucrados la pertinencia de las interpretaciones realizadas.

La observación de las implicaciones del cambio tecnológico en la Licenciatura en Comunicación de la Universidad Juárez Autónoma de Tabasco que se reporta en este documento, considera dos ejes de análisis: el institucional y el personal. El primero comprende los cambios y acciones que de manera objetiva han quedado registrados en documentos institucionales (planes y programas de estudio) y algunas consideraciones en el equipamiento de espacios para la formación. El eje personal, considera las acciones, y transformaciones que, a nivel personal, paulatino y natural, el cambio 
tecnológico ha implicado en los docentes como agentes de los procesos formativos que tienen lugar en la licenciatura.

Cambio en el currículo de la Licenciatura en Comunicación. El impacto en la era digital

El Plan de Estudios de la Licenciatura en Comunicación registró desde su incorporación a la oferta educativa de la institución, en 1991, tres reestructuraciones y un ajuste de créditos; momentos en los que las modificaciones reflejadas en el contexto de la disciplina y la profesión han podido analizarse y constituirse en argumentos para la reorientación de perfiles y la inclusión de nuevas asignaturas. Se realizó un análisis curricular comparado y sobre esta base se desarrolló el eje que se denominó: institucional. En él se identificaron los tres impactos en el currículo correspondientes a los momentos en que se modificó el plan, lo cual fue provocado en gran medida por el cambio tecnológico.

El Plan de Estudios fundacional (1991) de la Universidad Juárez Autónoma de Tabasco (UJAT) fue producto de una demanda social para profesionalizar la actividad que se venía realizando en los medios de comunicación locales, además de proveer elementos para el conocimiento, comprensión y reflexión sobre la relevancia de la comunicación en los diversos ámbitos sociales. Su estructura apuntaba a incorporar la actividad comunicativa- desarrollada hasta entonces en la entidad con bases meramente instrumentales-, a un campo de estudio académico, de reflexión y de sistematización de procesos.

El conocimiento tecnológico implicado comprendía procesos e instrumentos de producción visual, editorial, radiofónica y televisiva analógicos. En consecuencia, las asignaturas que procuraban el dominio tecnológico 
necesario para la formación del profesional de la comunicación versaban sobre técnicas e instrumentos producción radiofónica, televisiva de señal electromagnética, así como producción de comunicación impresa en diferentes formatos. No figuraban asignaturas ni contenidos que proyectaran la aplicación de tecnologías digitales.

Primer impacto: De la era de comunicación de masas, a la información multimedia y en red. El Plan de Estudios 2004, esgrime como argumentos rectores del cambio curricular propuesto por la institución, la necesidad de flexibilizar los sistemas de créditos, procurar pertinencia de las licenciaturas con los requerimientos de la sociedad e incorporar los avances científicos y tecnológicos que demandan los sectores para alcanzar el desarrollo integral del estado, entre otros (UJAT, 2004, p.18). De manera particular la evaluación al Plan de Estudios de la Licenciatura en Comunicación 1991, acusa que «la carencia de espacios y tecnología aplicada a la práctica no permiten una formación acorde a los nuevos tiempos» (UJAT, 2004 p.28). El documento, focaliza necesidades del campo de la comunicación acordes con la «etapa de tránsito de la era de comunicación de masas a una nueva era de la información multimedia y en red (...). Tránsito de la sociedad de los medios masivos a la sociedad de la información como contexto básico para interpretar las nuevas formas de comunicación» (UJAT,2004, p.46).

En consecuencia, la propuesta de formación emanada de la primera reestructuración, incorpora seis asignaturas que inician al estudiante en el manejo de tecnologías digitales (ver tabla 1).

Plan de Implicaciones del cambio Características

Estudios tecnológico en el currículo 


\begin{tabular}{lll}
\hline $1991-$ & Formación orientada a medios & Tecnología para de medios \\
2004 & masivos con tecnologías electrónicos radio, televisión, \\
analógicas. & diseño y manejo de imágenes \\
& con base analógica formatos \\
& tradicionales.
\end{tabular}

2004- Ajustes orientados a la impulsar Incorporación de asignaturas 2010 la transición de lo analógico a lo destinadas al manejo de digital con carácter tecnologías digitales específicas predominantemente instrumental. de apoyo para la formación del estudiante y de aplicación concreta en áreas de la profesión.

Asignaturas obligatorias incorporadas:

De carácter teórico

- Nuevas tecnologías en comunicación e información.

- Tecnologías de la información y comunicación en educación.

Asignaturas prácticas

- Herramientas de computación

- Informática aplicada a la investigación

- Periodismo en línea

- Diseño gráfico por 
computadora

2010- Cambio en la concepción de los El componente tecnológico 2017 escenarios de desarrollo de la implicado en la formación se profesión y de las demandas incorpora de forma transversal en para el Licenciado en las asignaturas del Plan de Comunicación.

Estudios.

Se reconfigura el perfil de En los contenidos de las egreso de un profesional, que asignaturas y su abordaje, el aspira a ser autoadaptativo y cambio tecnológico es inminente. autogestivo.

2017 La implicación se asume en su Incorporación transversal de dimensión socio cultural. contenidos con proyección hacia El ajuste curricular adopta un la interpretación y comprensión carácter prospectivo y holístico de los fenómenos socioque comprende el estudio y la tecnológicos. Los programas de reflexión de los procesos las asignaturas asumen la tecnológicos y los fenómenos transformación de contextos y emergentes derivados de la procesos sociales movilizados transformación de los por la innovación tecnológica. escenarios de sociales y por Se delinean y apuntalan saberes, tanto comunicativos. habilidades y competencias para soportar los procesos de

Se propone la formación de un apropiación y estudio de los profesional bricolaje. embates tecnológicos. Se privilegia la formación de capacidades que faculten el 
desempeño profesional en escenarios cambiantes.

Asignaturas que atienden expresamente el espectro tecnológico en la profesión y la sociedad:

De carácter Teórico:

- Cultura digital

- Estética de la comunicación

- Arquitectura de la comunicación.

De carácter práctico:

- Formatos digitales

-Periodismo en hipermedia

- Guion y narrativas audiovisuales

Tabla 1: Implicaciones del cambio tecnológico en planes de estudio de la Licenciatura en Comunicación de la UJAT (Fuente: elaboración propia).

Segundo impacto. Nuevos oficios, nuevas demandas, la necesidad de tecnologizar los campos. La segunda reestructuración, que entró en operación en 2010, problematiza el estudio del campo de la comunicación considerando, entre otros aspectos, la disolución de las fronteras del campo ante la evidente omnipresencia del fenómeno comunicativo en la vida social y la visión pluridisciplinaria con la que se aborda. 
La comunicación se ha generalizado, extendido, especializado, profesionalizado y abierto a nuevos modelos y esquemas; se han constituido oficios nuevos [...], amplios sectores que demandan especialistas del campo, como los movimientos sociales, las organizaciones no gubernamentales, áreas específicas del sector oficial como las de salud, cultura, recreación, deporte y otras (Universidad Juárez Autónoma de Tabasco, 2010, p.24).

Otro aspecto identificado como parte de la problemática del campo de estudio y profesionalización de la comunicación es la innegable brecha tecnológica entre los ámbitos de desempeño profesional de los licenciados en comunicación y su formación, especialmente en materia tecnológica.

Existe un desfase entre los aprendizajes áulicos y lo que se demanda del comunicólogo en el mercado laboral: conocimientos sobre nuevas tecnologías (digitalización de la información), habilidades para la resolución de problemas, etc., ya que las instituciones educativas no responden a los constantes cambios en la sociedad de la información, sobre todo debido a las estructuras tradicionales inflexibles; de hecho, existe plena conciencia de que es imposible la simultaneidad entre las constantes transformaciones del mundo debido al desarrollo científico y tecnológico, las posibilidades formativas de las instituciones de educación superior (UJAT, 2010, p.17).

Consecuente con el análisis de las problemáticas del campo, el Plan de Estudios 2010 registra un cambio sustantivo en su currículo: disgrega la formación de los egresados en cuatro áreas terminales, asociadas con cuatro de los siete campos disciplinares que convergen en la formación del comunicólogo: Publicidad y Diseño, Divulgación, Comunicación Organizacional y Comunicación Emergente. Las asignaturas optativas se concentran en los 
últimos dos ciclos, con el propósito de enfatizar en la profesionalización específica de acuerdo al campo elegido por el estudiante

El fortalecimiento de un perfil con énfasis profesional específico, responde a las características disciplinares, a las necesidades contemporáneas y a las preferencias de formación profesional de los estudiantes, además, pretende atender el aumento de la necesidad de profesionales cualificados en el cada vez más diverso campo de la comunicación, emulando la configuración desagregada de las titulaciones más recurrentes para el desempeño profesional: Comunicación Organizacional; Publicidad y Diseño, y Divulgación. A estos tres campos se suma el de la comunicación emergente que se presenta como un escenario de prácticas alternativas para el profesional de la comunicación frente a los nuevos escenarios y las demandas sociales.

La conformación del campo disciplinar de Comunicación Emergente, se orientaría a la formación del profesional de la comunicación capaz de atender situaciones emergentes en la sociedad, que trastocan la dinámica cotidiana y ameritan una intervención oportuna y razonada del profesional de la comunicación en un escenario transdisciplinar. Esta perspectiva de reciente consideración para las profesiones, representa una propuesta para redimensionar el papel de los profesionales en una sociedad cambiante, impredecible, de riesgo (Beck, 2004). En este sentido la adaptabilidad de las capacidades y habilidades de los profesionales representarán la condición necesaria para hacer frente a diversos escenarios de la actividad humana (UJAT, 2010, p. 49).

Las implicaciones del cambio tecnológico en Plan de Estudios 2010, adoptan de este modo un carácter más discreto; la revisión del currículo permite identificar, únicamente, la sustitución de la asignatura Periodismo en Línea por 
la de Ciberperiodismo, así como la desaparición de dos asignaturas que en su denominación aludían directamente al componente tecnológico en sus contenidos: Informática Aplicada a la Investigación y Tecnologías de la Información y Comunicación en Educación. Sin embargo, los ajustes emblemáticos de este Plan de Estudios reflejan una concepción del campo profesional en donde el cambio tecnológico no se traduce únicamente la necesidad de incorporación física de la tecnología, sino permea de forma integral en los procesos y escenarios comunicativos de la sociedad, por lo que estos ajustes en la formación se asumen de forma transversal en los contenidos y abordajes de las asignaturas. La tecnología se incorpora como algo inherente a las actividades del campo profesional.

En relativamente pocos años, la comunicación se ha generalizado, extendido, especializado, profesionalizado y abierto a nuevos modelos y esquemas; se han constituido oficios nuevos [....] y se ha abierto a nuevos medios. En otras palabras, ha segmentado su discurso, en la medida en que se ha complicado por el hecho de la multiplicación de los emisores, de los mensajes, de los medios, de los públicos, por lo que los parámetros para elaborar estrategias de comunicación requieren cada vez de mayores y complejas acciones (UJAT, 2010, p.24).

Ante la inminente reconfiguración de la profesión de la comunicación, los nuevos escenarios de desarrollo y las consecuentes exigencias para el desempeño de los profesionales del campo, derivadas de cambio constante de las sociedades por artífice tecnológico, el Plan de Estudios 2010 de la licenciatura en comunicación apuesta por la formación de un profesional 
autoadaptativo. Esta condición implica una formación flexible que permita no sólo el desarrollo de competencias genéricas de la profesión, sino de habilidades para adaptarse a situaciones diversas y cumplir con las demandas emanadas de las circunstancias.

Tercer impacto. Nuevas formas de interacción y nuevas funciones, el profesional bricolaje. El proceso de reestructuración, que dio paso a Plan de Estudios 2017 reconoce los retos que afronta la profesionalización de la comunicación ante la rotunda transformación de múltiples procesos sociales, entre ellos los procesos, formas y medios de comunicación. El espectro del cambio tecnológico no trastoca solo los derroteros de la profesión, «es la misma definición de sociedad la que ahora está en juego» (UJAT, 2017, p. 43). La convergencia comunicativa es el contexto para interpretar las nuevas formas de interacción, así como las nuevas funciones, los nuevos usos de los medios de comunicación y por tanto de la profesión.

«El futuro para el estudio de la comunicación se asoma retador. El profesional del campo debe tener las competencias [...] para gestionar procesos de comunicación en escenarios cada vez más complejos, aprovechando los nuevos medios y plataformas como las redes sociales y la telefonía móvil» (UJAT, 2017, p. 39).

La base de los cambios en el currículo, en la versión del Plan de Estudios 2017, está en la concepción de las transformaciones gestadas en la sociedad y la cultura digital, "presenta la urgente necesidad de un conocimiento descentralizado y diversificado y una alta demanda de aprendizajes continuos y masivos, a fin de generar nuevos conocimientos que contribuyan a interpretar y transformar la realidad» (UJAT, 2017, p. 44). 
La comprensión de la condición socio-tecnológica cambiante de los contextos y problemáticas imbricados en los fenómenos comunicativos, se constituye en el itinerario al que apunta la formación del licenciado en comunicación. En ese sentido la propuesta de la formación de la UJAT transforma su aspiración de un profesional autoadaptativo a un profesional bricolaje, cuando señala que para hacer frente a los requerimientos que demandan los nuevos medios, lenguajes y situaciones emergentes de comunicación, se necesita de la formación de un profesional innovador y creativo. que dé respuesta a los cambios del entorno.

Las tendencias apuntan a un bricolaje, entendido como aquel que utiliza los instrumentos que encuentra a su disposición alrededor suyo, que están ya ahí, que no habían sido concebidos especialmente con vistas a la operación para la que se hace que sirvan, y a la que se los intenta adaptar por medio de tanteos, no dudando en cambiarlos cada vez que parezca necesario hacerlo. Con este fundamento se abre un horizonte de atención a los lenguajes humanos en interacción con la tecnología, lo que incluye otros sistemas, otros códigos y otras reglas sociales (UJAT, 2017, p.45).

La revisión de los programas del Plan de Estudios 2017 y sus contenidos, destacan la inclusión de manera holística y transversal, del abordaje y reflexión de los escenarios digitales movilizados por el cambio tecnológico. Las áreas de desempeño de la profesión, así como los paradigmas epistémicos y metodológicos que les explican, discurren en las transformaciones inminentes de los escenarios comunicativos, sus lenguajes, canales, actores y funciones.

La malla curricular denota la transformación del campo de divulgación en la línea de Comunicación convergente a fin de implicar el encuentro entre las formas de comunicación tradicional con los formatos digitales. Las asignaturas no aluden a medios específicos (por ejemplo: producción radiofónica vs. 
producción sonora, producción televisiva vs producción audiovisual), más bien su designación está orientada a dar cabida a múltiples medios y formatos; rasgo que parece responder a la intención de formación del perfil bricolaje. Así mismo es notable la inclusión de asignaturas encaminadas a la revisión y comprensión de los escenarios contingentes que se perfilan para la comunicación y algunas otras que replantean atributos de los procesos y fenómenos comunicativos que toman vigencia con la emergencia de contextos comunicativos convergentes.

La implicación más significativa del espectro tecnológico en el currículo es producto del entendimiento de la transformación del panorama comunicativo en las sociedades, del campo profesional y en consecuencia, la necesidad de asumir estrategias formativas que den respuesta a las exigencias de los nuevos tiempos de la comunicación; visión que se incorporará como parte fundamental del espíritu del Plan de Estudios de la Licenciatura en Comunicación de forma paulatina.

El segundo eje de análisis que aquí se ofrece, involucra a los sujetos que se constituyen como referentes en la transformación de la formación del profesional de la comunicación. Este apartado convoca la mirada de docentes (D1, D2, D3 y D4) que, con base en el tránsito en al menos tres de los planes de estudio de la licenciatura en comunicación de la institución, ofrecen sus puntos de vista en torno a los cambios en la profesión y las estrategias curriculares y pedagógicas implementadas para su atención en la licenciatura.

Impacto de la era digital en la formación del comunicólogo: la mirada docente 
Uno de los elementos fundamentales en la formación del estudiante es sin duda el docente. En todos los niveles educativos el profesor se enfrenta a la exigencia de utilizar una serie de recursos tecnológicos en el aula, lo cual puede ser concebido como un reto, pero también como un problema. En las disciplinas sin excepción, la tecnología ha tenido impacto, en mayor o menor medida, y se ha hecho necesario incorporarla a los procesos educativos. En el caso de Comunicación como profesión, el impacto es muy significativo, dado que son las tecnologías de información y comunicación el principal medio con que cuenta el comunicólogo para el desarrollo de su profesión.

En los docentes entrevistados se buscaba conocer su percepción respecto al impacto que la era digital ha tenido en la formación del comunicólogo, así como la manera en que la licenciatura ha respondido a las implicaciones del cambio tecnológico. Conforme a sus respuestas, se pudieron distinguir algunos patrones de regularidad en ambos sentidos, los cuales se exponen a continuación.

Primer impacto: La brecha tecnológica y el currículo. Los docentes concuerdan en que siempre ha existido una brecha tecnológica entre lo que se enseña en la escuela y lo que se vive en el mundo real (espacio laboral). Las universidades carecen de los espacios y recursos tecnológicos suficientes para emular el ámbito profesional, el mundo va a una velocidad mucho mayor que las estructuras institucionales y por ende curriculares, haciendo difícil la convergencia de todas.

"Las instituciones formadoras en general, pero en especial las públicas mantienen una brecha tecnológica con los ámbitos laborales ... Es un asunto viejo, aun en el trabajo con tecnologías analógicas, el reclamo de 
los empleadores tenía que ver con que los egresados tenían que terminar de capacitarse en el uso de equipos ya en el trabajo" (D1).

Los docentes consideran que la Comunicación como profesión tienen una base donde el componente tecnológico es vital para afianzar su perfil en las diversas condiciones tecnológicas. En virtud de esto el docente debe mantenerse en contacto permanente con las culturas de trabajo externas donde se desempeña el profesional de la Comunicación. Sin embargo, y pese a ser un elemento esencial, la tecnología por sí misma no es la panacea de todos los problemas, ante la imposibilidad de cambiar la realidad, el docente ha de imbuir en el estudiante una actitud de actualización constante.

(...) "la universidad, debe sentar las bases para que laboralmente el mismo egresado busque una actualización abierta en lo tecnológico" (D2).

Segundo impacto. Nuevas oportunidades laborales. La situación tecnológica como condicionante de la estructura y orientación de la formación del profesional de la comunicación desde la óptica de los docentes, trasgrede la noción instrumentalista y anida en asuntos más complejos y sustantivos, como la concepción misma de las prácticas y áreas de desarrollo de la profesión, la re-significación de lenguajes, el conocimiento de la transformación de las audiencias y la formación ética del profesional. La diversificación de las tareas y su dominio trae aparejadas nuevas alternativas de trabajo que replantean nuevos perfiles, idea que es abrazada por los docentes, quienes toman conciencia de su papel en este desafío. 
"La propia tecnología ahora mismo ha orientado los espacios laborales de la comunicación a nuevas oportunidades laborales (...) hablo por ejemplo de los youtubers, los twitteros, blogueros; todos estos líderes de opinión en tecnología que, más allá de tener un liderazgo, requieren también de una formación profesional (...). No se trata nada más un hábil adiestramiento en recursos tecnológicos, tiene que tener un fondo que forme al estudiante en la idea de que la tecnología va ser bien empleada (D2).

Los profesores están convencidos que el contexto está determinando el derrotero que lleva el plan, ya que condiciona hasta cierto punto los contenidos de las asignaturas

"El cambio tecnológico impacta la sociedad en general y así se asume en la formación de los comunicólogos, no como algo exclusivo de la profesión sino del contexto donde se forman y se desempeñaran, por lo que básicamente se ha transformado la forma en cómo percibimos y entendemos a la sociedad" (D4).

Frente a la presencia de estos cambios y nuevas necesidades se hicieron los ajustes curriculares con los que se pretendió dar respuesta a los mismos. En la mirada de los profesores entrevistados el Plan de Estudios de la Licenciatura en Comunicación en sus diferentes reestructuraciones ha tratado de responder con pertinencia a las implicaciones del cambio tecnológico tanto en la profesión como en la sociedad fortaleciendo el análisis de los fenómenos sociales y comunicativos. Sin embargo, el espectro de cambio digital e innovación 
tecnológica de la profesión alcanza las dinámicas áulicas, en las que el papel del docente es señalado como estratégico.

\begin{abstract}
"Ahora podemos decir que la tecnología digital es la que está orientando el perfil de la carrera (...) Pero creo que todavía tiene algunas ausencias, algunos vacíos, y me parece que no necesariamente del Plan de Estudios como tal sino de las condiciones institucionales que le acompañan, por ejemplo, la actualización del docente" (D3).
\end{abstract}

Si bien consideran que el impacto en los planes y programas de estudio ha sido obvio y trascendente, no ha sido menos en la docencia, que se vio implicada en un dejo constante de transformaciones que inciden en la formación y capacitación permanente. El trabajo del docente representa un punto clave para la ejecución de un programa de estudios, ya que la tecnología por sí misma no es garantía de éxito en el proceso de aprendizaje.

"La tecnología, forma un papel vital en comunicación, pero no es ella por sí misma la fortaleza de un Plan de Estudios; esta estructura fuerte, vital en los planes de estudio de comunicación, debe estar acompañada por una formación de sentido crítico, de reflexión y que la opere, si no, esto se nos va de las manos" (D1).

Sin embargo, no todos los docentes están dispuestos a asumir el cambio que conlleva la habilitación de la tecnología en el aula, ellos mismos confiesan que existe mucha resistencia y que ésta se maneja a discreción por parte del docente que imparte alguna asignatura. 
(...) "desgraciadamente (el docente) se convierten también en un contrapeso que hace más lenta la llegada a las aulas de los reclamos del campo laboral" (D4).

El reconocimiento de la reconfiguración del campo profesional de la comunicación es una realidad visible tanto en los documentos que conducen la formación de los licenciados en comunicación en la Universidad Juárez Autónoma de Tabasco, como para docentes quienes se constituyen en artífices de dicha formación. El cambio tecnológico es señalado como un motor principal de esta reconfiguración y en consecuencia un factor determinante en la toma de decisiones en la transformaciones de los planes de estudio; pese a la reconocida imposibilidad de las procesos formativos de marchar a la par de los acelerados procesos de innovación tecnológica, las institución ha trazado estrategias formativas que perfilen a un profesional capaz de atender las demandas de los cambiantes escenarios de desempeño comunicativo en los que está llamado a intervenir. La clave, que el análisis realizado nos permite dilucidar, consiste en no sucumbir en la carrera tecnológica sino afrontarla apuntalando la formación de capacidades alternas, que faculten procesos de apropiación y actualización tecnológica, flexibles y contextualizados.

\section{Conclusiones}

El cambio tecnológico se erige como un reto constante al que la educación superior debe atender. No se trata de un asunto disciplinar sino de una condición irrefrenable e inmanente a las sociedades; sin embargo, comprender y dimensionar las transformaciones particulares en cada campo 
de acción y desempeño faculta el sostenimiento de la pertinencia social de la formación profesional.

El campo de la comunicación, inexorablemente unido a la tecnología, transforma sus prácticas, sus escenarios, sus alcances, como consecuencia del cambio tecnológico, así lo reconocen los docentes entrevistados, visión que concuerda con la denunciada emergencia de nuevos lenguajes y soportes mediáticos (García y Román, 2018; López, 2012), de nuevos perfiles y puestos laborales (Benítez, 2007; Scolari et al, 2008; Soler y Aguilar, 2009). Se trata del ensanchamiento y la diversificación del ya antes basto campo de desempeño profesional de la comunicación, que reclama replantear la formación de sus profesionales.

Las revisiones de los planes de estudio de la UJAT para la licenciatura en comunicación documentan de manera parcial, las transformaciones percibidas en la profesión y las formas en las que institucionalmente se plantea hacer frente a las mismas: con incorporación de nuevos contenidos, pero sobre todo con la propuesta de redimensionar la profesión en sus diversos escenarios lo que queda de manifiesto en la adecuación de sus principios y orientación (UJAT, 2004; UJAT, 2010; UJAT,2017).

Frente a la aparente relevancia de rezago de equipamiento tecnológico de la universidad pública, se ubican dos asuntos determinantes para atender las implicaciones del incontenible cambio tecnológico: el reconocimiento de la reconfiguración del campo profesional de la comunicación y el reclamo de desarrollo de habilidades que faculten al profesional a transitar en escenarios de cambio y actualización constante provocado por la hipermedia (Sévigny \& Flynn, 2011). 
En términos generales se puede pensar que las implicaciones del cambio tecnológico en la formación de profesionales, encontrarán un catalizador, no en la actualización tecnológica, sino en la actualización docente sobre la que descansa en gran medida, la orientación y comprensión de las exigencias y derroteros de la profesión. Esta idea es congruente con lo que afirman autores (Sévigny \& Flynn, 2011; Ventura, et al, 2018) sobre la necesidad de compensar el conocimiento adquirido en la academia con las habilidades y capacidades demandadas en el sector laboral digital.

Conscientes de este desfase se ha propuesto el Marco Europeo para Organizaciones Educativas Digitalmente Competentes (DigCompOrg) mismo que puede utilizarse como guía para el proceso de autorreflexión en su avance hacia una amplia integración y un despliegue eficaz de tecnologías de aprendizaje digital y para la adopción eficaz de las mismas por parte de las organizaciones educativas ADECCO (2016).

\section{Bibliografía}

- ADECCO, (2016). Informe sobre el futuro del trabajo en España. Madrid: Adecco. Recuperado de https://loentiendo.com/wpcontent/uploads/2016/02/informe-Adecco-futuro-del-trabajo.pdf

- Álvarez-Flores, E., Núñez-Gómez, P. y Olivares-Santamarina, J. (2018). Perfiles profesionales y salidas laborales para graduados en Publicidad y Relaciones públicas: de la especialización a la hibridación. El profesional de la información, 27(1), 136-147. Recuperado de https://bit.ly/2OUi95h

- Anderson, C., Bell, E. \& Shirky, C. (2017). Post Industrial Journalism: Adapting to the Present. Columbia Journalism School, 1-119. https://doi.org/10.7916/D8N01JS7 
- Bazeley, P. (2013). Qualitative data analysis. Practical Strategies [Análisis cualitativo de datos. Estrategias prácticas]. Estados Unidos de América: SAGE.

- Beck, U. (2004). La sociedad del riesgo: Hacia una nueva modernidad. Barcelona, España: Paidós.

- Benítez, N. (2007). El documentalista audiovisual: perfil y tareas del profesional en la era de la información digital. Ponencia presentada en las IX Jornadas de Gestión de la Información, Asociación Española de Documentación e Información. Recuperado de. http://eprints.rclis.org/10849/1/Noelia Ramirez.pdf

- Brandtzaeg, P., Luders, M., Spangenberg, J., Rath-Wiggins, L. \& Folstad, A. (2015). Emerging journalistic verification practices concerning social media. Journalism practice, 10(3), 323-342.

https://doi.org/10.1080/17512786.2015.1020331

- Brewer, L. (2013). Enhancing youth employability: ¿What? Why? ¿and How? Guide to core work skills [Mejorar la empleabilidad de los jóvenes: ¿Qué? ¿por qué? ¿Y cómo? Guía de habilidades básicas de trabajo]. International Labour Organization, Skills and Employability Department. Recuperado de https://cutt.ly/dym93IF

- Carrillo-Durán, M. y Rodríguez-Silgado, A. (2018). El ecosistema programación. La nueva publicidad digital que conecta datos con personas. El profesional de la información, 27(1), 195-201. https://doi.org/10.3145/epi.2018.ene.18

- Cheney, G. \& Ashcraft, K. (2007). Considering "The Professional" in communication studies: implications for theory and research within and beyond the boundaries of organizational communication. Communication 
Theory, $\quad 17(2), \quad 146-175 . \quad$ https://doi.org/10.1111/j.14682885.2007.00290.x

- Cho, M., Furey, L. \& Mohr, T. (2016). Communicating corporate, social responsibility on social media: strategies, stakeholders, and public engagement on corporate Facebook. Business and Professional $\begin{array}{lll}\text { Communication } \quad \text { Quarterly, } & \text { 80(1), } & \text { 1-18. }\end{array}$ https://doi.org/10.1177/2329490616663708

- Creswell, J. (2015). A concise introduction to mixed methods research [Una introducción concisa a los métodos de investigación mixtos]. Estados Unidos de América: SAGE.

- DuFrene, D. \& Lehman, C. (2014). Navigation change: employee communication in times of instability. Business and Professional Communication Quarterly, $77(4)$, 1-10.

\section{https://doi.org/10.1177/2329490614544736}

- Edwards, R. \& Holland, J. (2013). What is qualitative interviewing? [¿Qué es una entrevista cualitativa?]. Reino Unido: Bloomsbury.

- Elorriaga-Illera, A., Usin-Enales, S. y García-Azpuru, A. (2018). Evolución del community manager en las pymes del País Vasco y Navarra. El profesional de la información, 27(3), 651-658. Recuperado de https://bit.ly/2KdDbXW

- García, A. y Román, M. (2018). Sobre los nuevos modelos de negocio en las actividades radiofónicas. Revista de la Asociación Española de Investigación de la Comunicación, 4(7), 40-45. Recuperado de http://www.revistaeic.eu/index.php/raeic/article/view/85 
- García-Aretio, L. (2019). Necesidad de una educación digital en un mundo digital. RIED. Revista Iberoamericana de Educación a Distancia, 22(2), 9-22. Recuperado de https://bit.ly/33cY8tt

- Jameson, D. (2014). Crossing public-private and personal-professional boundaries: how changes in technology may affect CEOs Communication. Business and Professional Communication Quarterly, 77(1), 7 -30. https://doi.org/10.1177/2329490613517133

- Jódar, J. (2010). La era digital: nuevos medios, nuevos usuarios y nuevos profesionales. Razón y palabra, (71), 1-11. Recuperado de http://www.razonypalabra.org.mx/N/N71/VARIA/29\%20JODAR REVISA DO.pdf

- Kerr, A. \& Kelleher, J. (2015). The recruitment of passion and community in the service of capital: community manager in the digital games industry. Critical Studies in Media Communication, 32(3), 177-192. https://doi.org/10.1080/15295036.2015.1045005

- López, X. (2012). La formación de los periodistas para los entornos digitales actuales. Revista de Comunicación, 11(), 178-195. Recuperado de https://revistadecomunicacion.com/es/articulos/2012/Art178-195.html

- López-García, X., Rodríguez-Vázquez, A. y Pereira-Fariña, X. (2017). Competencias tecnológicas y nuevos perfiles profesionales: desafíos del periodismo actual. Revista Comunicar, 25(53), 81-90.

https://doi.org/10.3916/C53-2017-08

- Martínez-Sala, A. y Campillo-Alhama, C. (2018). La gestión de las redes sociales turísticas desde la perspectiva de las relaciones públicas 2.0: la importancia del diálogo. Revista Internacional de Relaciones Públicas, 8(16), 5-26. Recuperado de 
http://revistarelacionespublicas.uma.es/index.php/revrrpp/article/view/55 $\underline{5 / 30}$

- Meso, K. (2003). La formación del periodista digital. Revista Latinoamericana de Comunicación CHASQUI, (84), 1-11. Recuperado de https://revistachasqui.org/index.php/chasqui/article/view/1497/1525

- Miquel, S., López, L. y Gil, S. (2018). Radiografía de las consultoras de comunicación en España: perfil profesional, estructura y actividad. Revista Latina de Comunicación Social, 73, 478-503. https://doi.org/10.4185/RLCS-2018-1266 Molina, J. y López, C. (2018). Mutación digital. Claves para el desarrollo de la docencia de la comunicación corporativa en el ámbito de la educación líquida. Telos. Revista de Pensamiento, Sociedad y Tecnología, (101), 63-71. Recuperado de https://cutt.ly/5ymBz2W

- Muñoz-de-la-Peña-Costero, P., Meana-Alonso, S. y Sáez-Carreras, S. (2014). Cinco años de experiencia digital en los Servicios Informativos de TVE: una nueva gestión de contenidos. El profesional de la información, 23(1), 72-79. https://doi.org/10.3145/epi.2014.ene.09

- Ortega, Á., Rendón, L. y Ortega, J. (2017). La profesionalización del community manager: claves para una formación especializada de posgrado. Revista Lasallista de investigación, 14(1), 217-232.

https://doi.org/10.22507/rli.v14n1a19

- Porter, J. (2017). Professional communication as phatic: from classical Eunoia to personal artificial intelligence. Business and Professional Communication Quarterly, $80(2)$, 1-20. https://doi.org/10.1177/2329490616671708 
- Ripoll-Mont, S., Tolosa-Robledo, L. (2009). El documentalista de programas de televisión: horizontes profesionales. El profesional de la información, 18(3), 341-347. Recuperado de. http://eprints.rclis.org/19426/1/El\%20Profesional\%20de\%20la\%20Inform acion2009.pdf

- Rowley, J. (2008). ¿Just another channel? Marketing communications in e-business. Marketing Intelligence \& Planning, 2(1), 24 -31. https://doi.org/10.1108/02634500410516896

- Scolari, C., Mico, J., Navarro, H. y Pardo, H. (2008). El periodista polivalente. Transformaciones en el perfil del periodista a partir de la digitalización de los medios audiovisuales catalanes. Zer Revista de Estudios de Comunicación, 13(25), 37-60. Recuperado de https://www.ehu.eus/ojs/index.php/Zer/article/view/3572/3200

- Sévigny, A. \& Flynn, T. (2011). A reflection on the evolution of the field of professional communication. Journal of Professional Communication, 1(1), 3-14. Recuperado de. https://mulpress.mcmaster.ca/jpc/article/view/79/34

- Silva-Robles, C. (2016). Perfil del community manager en las agencias de publicidad y relaciones públicas de España. El profesional de la información, 25(2), 237-245. Recuperado de https://bit.ly/2Yjl3DE

- Soler, P. y Aguilar, M. (2009). Formación de los comunicadores en la era digital. Madrid, Fundación Alternativas. Recuperado de https://cutt.ly/Rym9MfH

- Soler-Adillon, J., Sora, C., Freixa, P. y Ribas, J. (2016). Perfil del profesional de la comunicación interactiva: fundamentos, actualidad y 
perspectivas. El profesional de la información, 25(2), 196-208. https://doi.org/10.3145/epi.2016.mar.06

- Universidad Juárez Autónoma de Tabasco. (UJAT). (2004). Plan de Estudios 2004 Licenciatura en Comunicación. [Documento oficial]. México: Universidad Juárez Autónoma de Tabasco.

- Universidad Juárez Autónoma de Tabasco. (UJAT). (2010). Plan de Estudios 2010 de la Licenciatura en Comunicación "[Documento oficial] México: Universidad Juárez Autónoma de Tabasco.

- Universidad Juárez Autónoma de Tabasco. (UJAT). (2017). Plan de Estudios 2017 de la Licenciatura en Comunicación [Documento oficial] México: Universidad Juárez Autónoma de Tabasco

- Ventura, R., Roca-Cuberes, C. \& Corral-Rodríguez, A. (2018). Interactive Digital Communication: assessment of professional, teachers and students in the area of communication on academic competences and professional profiles. Revista Latina de Comunicación Social, 73, 331-351. Recuperado de. http://www.revistalatinacs.org/073paper/1258/17es.html

- Yin, Robert. (2012). Case study research. Design and methods [Investigación de casos de estudio. Diseño y métodos]. Estados Unidos de América: SAGE. 